\title{
Extension of the Finite Integration Technique including dynamic mesh refinement and its application to self-consistent beam dynamics simulations
}

\author{
Sascha M. Schnepp* \\ Graduate School of Computational Engineering, Technische Universität Darmstadt, \\ Dolivostraße 15, 64293 Darmstadt, Germany \\ Erion Gjonaj ${ }^{\dagger}$ and Thomas Weiland ${ }^{\ddagger}$ \\ Institut für Theorie Elektromagnetischer Felder, TEMF, Technische Universität Darmstadt, \\ Schlossgartenstraße 8, 64289 Darmstadt, Germany \\ (Received 27 June 2011; published 20 January 2012)
}

\begin{abstract}
An extension of the framework of the Finite Integration Technique (FIT) including dynamic and adaptive mesh refinement is presented. After recalling the standard formulation of the FIT, the proposed mesh adaptation procedure is described. Besides the linear interpolation approach, a novel interpolation technique based on specialized spline functions for approximating the discrete electromagnetic field solution during mesh adaptation is introduced. The standard FIT on a fixed mesh and the new adaptive approach are applied to a simulation test case with a known analytical solution. The numerical accuracy of the two methods is shown to be comparable. The dynamic mesh approach is, however, much more efficient. This is demonstrated with the full scale modeling of the complete rf gun at the Photo Injector Test Facility DESY Zeuthen (PITZ) on a single computer. Results of a detailed design study addressing the effects of individual components of the gun onto the beam emittance using a fully self-consistent approach are presented.
\end{abstract}

DOI: 10.1103/PhysRevSTAB.15.014401

PACS numbers: 02.70.Bf, 29.27.Bd, 41.75.Fr

\section{INTRODUCTION}

Memory consumption and CPU time represent the main limitations for large-scale electromagnetic field computations. This is especially the case for accelerator physics simulations involving self-consistent charged particle models based on the so-called particle-in-cell (PIC) method [1]. Simulations of this kind are an indispensable tool for the design and optimization of particle accelerators since they offer a full insight into the beam dynamics down to the particle level. This is especially important for the simulation of low-energy sections of an accelerator, where space-charge forces heavily influence the beam but also for, e.g., dark current or electron cloud simulations. Apart from the solution of Maxwell's equations on a discrete grid space, PIC simulations also include the self-consistent solution of the equations of motion. They take into account the two-way interaction of particles and electromagnetic fields including particle self-fields. Thus, they provide a full description of the accelerator structure including space-charge and wakefield effects [2].

\footnotetext{
*Author to whom correspondence should be addressed. schnepp@gsc.tu-darmstadt.de gjonaj@temf.tu-darmstadt.de

weiland@temf.tu-darmstadt.de
}

Published by the American Physical Society under the terms of the Creative Commons Attribution 3.0 License. Further distribution of this work must maintain attribution to the author(s) and the published article's title, journal citation, and DOI.
In this article we address the important problem of numerical efficiency of such beam dynamics simulations using the Finite Integration Technique (FIT). The FIT has been successfully applied for the simulation of a wide range of electromagnetic problems in accelerator physics $[3,4]$. We propose an extension of this method including dynamic mesh refinement in order to locally adjust the spatial grid resolution according to the dynamics of particles and fields. This leads to considerable savings in the overall number of computational degrees of freedom and, thus, reduces the computational burden in PIC simulations.

The article is organized as follows. After describing the governing equations in Sec. II, a brief review of the FIT on static grids is given in Sec. III, which also serves for introducing the notation. Section IV describes the dynamic mesh refinement procedures and Sec. V addresses specific issues of charged particle simulations on dynamically refined meshes. Section VI contains two applications. First, an example with a known analytical solution is considered for investigating accuracy and efficiency of the proposed method. After, results of a detailed design study of the PITZ photoinjector using self-consistent PIC simulations are presented. The achievements are summarized in Sec. VII.

\section{FORMULATION OF THE PHYSICAL PROBLEM}

The electromagnetic part of the physical problem considered is described by Maxwell's equations. Their integral form reads 


$$
\begin{gathered}
\int_{\partial S} \vec{H} \cdot d \vec{x}=\int_{S}\left(\frac{\partial}{\partial t} \vec{D}+\vec{J}\right) \cdot d \vec{A}, \\
\int_{\partial S} \vec{E} \cdot d \vec{x}=-\int_{S} \frac{\partial}{\partial t} \vec{B} \cdot d \vec{A}, \\
\int_{\partial V} \vec{D} \cdot d \vec{A}=\int_{V} \rho d V, \\
\int_{\partial V} \vec{B} \cdot d \vec{A}=0,
\end{gathered}
$$

where $S$ and $V$ denote arbitrary surfaces and volumes. The magnetic field strength is indicated by $\vec{H}, \vec{B}$ denotes the magnetic flux density, $\vec{E}$ the electric field strength, $\vec{D}$ the dielectric flux density, and $\vec{J}$ the electric current density. The electric and magnetic quantities are related according to the constitutive equations,

$$
\begin{aligned}
\vec{D} & =\epsilon \vec{E}, \\
\vec{B} & =\mu \vec{H} .
\end{aligned}
$$

For the linear, isotropic, nondispersive materials considered here, the permittivity $\epsilon$ and the permeability $\mu$ are material dependent constants. The conservation of charge follows from Ampère's law (1) and Gauss' law (3) in the form of the continuity equation,

$$
\frac{d}{d t} \int_{V} \rho d V+\int_{\partial V} \vec{J} \cdot d \vec{A}=0
$$

It reflects that a temporal change of the total charge, $Q=$ $\int_{V} \rho d V$, contained in a volume is caused only by a flow of electric current into or out of the volume.

The link between Maxwell's equations and the motion of charged particles is established by the Lorentz force,

$$
\vec{F}=q\left(\vec{E}+\frac{\vec{\nu}}{\gamma} \times \vec{B}\right)
$$

on the one hand, and Newton's law,

$$
\vec{F}=\frac{d}{d t} \vec{p}
$$

on the other hand. Above, $q$ is the charge of the particle, $\gamma$ is the relativistic factor, and $\vec{\nu}=\gamma \vec{v}$, with $\vec{v}$ the velocity of the particle. The mechanical momentum is denoted by $\vec{p}$. The equations of motion, thus, read

$$
\begin{gathered}
\frac{d}{d t} \vec{u}=\frac{\vec{\nu}}{\gamma}, \\
\frac{d}{d t} \vec{\nu}=\frac{q}{m}\left(\vec{E}+\frac{\vec{\nu}}{\gamma} \times \vec{B}\right),
\end{gathered}
$$

where $\vec{u}$ is the position vector and $m$ the particle mass.

\section{THE FINITE INTEGRATION FRAMEWORK}

For introducing the notation as well as for completeness, this section comprises a short review of the Finite Integration Technique. For the spatial discretization of Maxwell's equations, it utilizes a staggered, dual orthogonal grid doublet $(G, \tilde{G})$, which covers the domain of interest $\Omega$. The staggered grid arrangement is depicted in Fig. 1 . Throughout this article, we employ the FIT on Cartesian grids in three-dimensional space. The following discrete integral state variables are introduced:

$$
\begin{array}{lll}
\hat{e}:=\int_{c} \vec{E} \cdot d \vec{x}, & \hat{h}:=\int_{\tilde{c}} \vec{H} \cdot d \vec{x}, & \hat{\hat{b}}:=\int_{S} \vec{B} \cdot d \vec{A}, \\
\hat{d}:=\int_{\bar{S}} \vec{D} \cdot d \vec{A}, & \hat{\hat{j}}:=\int_{\tilde{S}} \vec{J} \cdot d \vec{A}, & q:=\int_{\tilde{V}} \rho d V,
\end{array}
$$

where $c$ and $S$ belong to the set of edges and faces of the primary grid (black), and $\tilde{c}, \tilde{S}$, and $\tilde{V}$ belong to the set of edges, faces, and volumes of its dual (gray). The quantities $\hat{e}$ and $\hat{h}$ represent grid voltages, whereas $\hat{\hat{b}}, \hat{\hat{d}}$, and $\hat{\hat{j}}$ are field fluxes on the faces of the grid.

Summing up the voltages $\hat{e}$ and $\hat{h}$ along the four edges enclosing one face yields a discrete but exact representation of Ampère's and Faraday's law, (1) and (2), in the form

$$
\begin{gathered}
\sum_{j=1}^{4} \pm \hat{h}_{j}=\frac{d}{d t} \hat{\hat{d}}+\hat{\hat{j}}, \\
\sum_{j=1}^{4} \pm \hat{e}_{j}=-\frac{d}{d t} \hat{\hat{b}}
\end{gathered}
$$

for every face of the computational grid. The orientation of the involved voltages $\hat{e}_{j}, \hat{h}_{j}$ with respect to the orientation of the loop integral in (1) and (2) determines the summation signs (see Fig. 1).

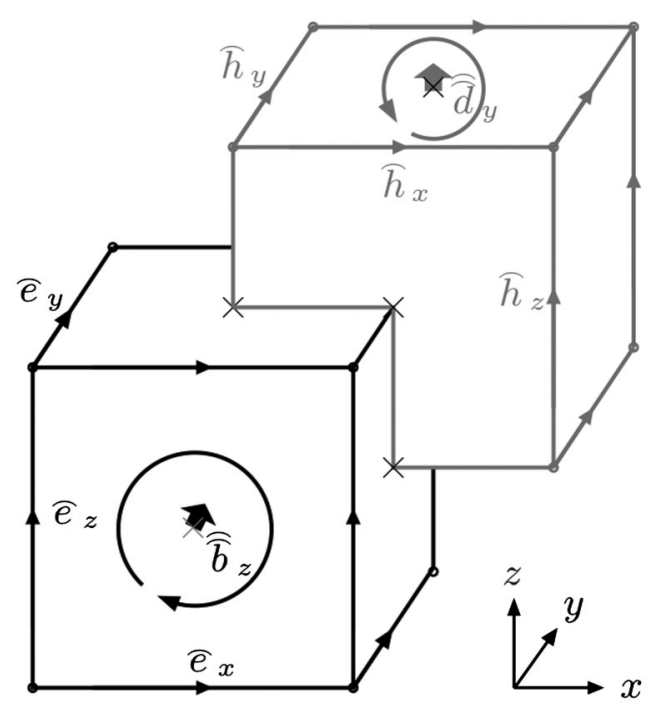

FIG. 1. Staggered grid doublet in FIT. The primary grid and its associated quantities are depicted in black, the dual grid in gray. 
The discrete Gauss' laws are obtained in a similar way by summing up the electric and magnetic flux variables $\hat{\hat{d}}$ and $\hat{b}$ defined on all faces of a cell. Fluxes pointing out of the cell are counted positive, incoming fluxes negative. This yields the algebraic set of equations,

$$
\begin{aligned}
& \sum_{j=1}^{6} \pm \hat{\hat{d}}_{j}=q, \\
& \sum_{j=1}^{6} \pm \hat{\hat{b}}_{j}=0,
\end{aligned}
$$

for every cell of $G$ and $\tilde{G}$ corresponding to the Gauss' law for electric and magnetic charges, respectively.

The constitutive equations (5) and (6) translate to

$$
\begin{aligned}
& \hat{\hat{d}}=M_{\epsilon} \hat{e}, \\
& \hat{\hat{b}}=M_{\mu} \hat{h},
\end{aligned}
$$

where $M_{\epsilon}$ and $M_{\mu}$ provide an appropriate mapping of grid voltages into fluxes [3].

Collecting the voltages and fluxes in the vectors $\hat{\mathbf{e}}, \hat{\mathbf{h}}, \hat{\hat{\mathbf{b}}}$, $\hat{\mathbf{d}}$, and $\hat{\hat{\mathbf{j}}}$ allows for stating (13)-(16) for the complete computational domain in the compact form,

$$
\begin{gathered}
\tilde{\mathbf{C}} \hat{\mathbf{h}}=-\frac{d}{d t} \hat{\hat{\mathbf{d}}}+\hat{\hat{\mathbf{j}}}, \\
\mathbf{C} \hat{\mathbf{e}}=-\frac{d}{d t} \hat{\hat{\mathbf{b}}}, \\
\tilde{\mathbf{S}} \hat{\mathbf{d}}=\mathbf{q}, \\
\mathbf{S} \hat{\hat{\mathbf{b}}}=\mathbf{0} .
\end{gathered}
$$

The summation signs are gathered in the matrices $\mathbf{C}, \tilde{\mathbf{C}}$ and $\mathbf{S}, \tilde{\mathbf{S}}$ [5]. These matrices are, hence, purely topological. They represent a discrete curl and divergence operator of the FIT and allow for expressing the Poincare lemmata in discrete space,

$$
\mathbf{S ~ C}=0, \quad \tilde{\mathbf{S}} \tilde{\mathbf{C}}=0, \quad \mathbf{C} \tilde{\mathbf{S}}^{\mathrm{T}}=0, \quad \tilde{\mathbf{C}} \mathbf{S}^{\mathrm{T}}=0 .
$$

This allows, e.g., for the derivation of a discrete continuity equation [cf. Eq. (7)] in the form

$$
\frac{d}{d t} \mathbf{q}+\tilde{\mathbf{S}} \hat{\mathbf{j}}=\mathbf{0} .
$$

Equations (22), (21), and (22) are called Maxwell grid equations. Typically, the leapfrog scheme is applied for their integration in time. Alternatively, one may apply the longitudinal-transverse split operator scheme introduced in $[6,7]$ for the time integration, which offers better numerical dispersion properties. The latter approach is referred to as LT-FIT standing for longitudinal-transverse-FIT.

\section{DYNAMIC MESH REFINEMENT}

We will restrict the discussion to conformal mesh refinements, which maintain the conformity and duality of the Cartesian grid doublet. A refined mesh is obtained by a sequence of bisections applied to a given mesh cell. The number of consecutive bisections of a cell is referred to as its refinement level $L$ (see Fig. 2). The refinement history of a cell can conveniently be represented as a binary tree, where the root node corresponds to the initial nonrefined cell. The tree leaves represent the current state of the refined mesh, where a leaf's level in the tree hierarchy corresponds to its refinement level $L$.

When mesh refinement is applied, the discrete quantities assigned to the primary and the dual grid need to be recomputed according to the modified mesh. In Fig. 3 the refinement of a primary grid cell and the subsequent arrangement of electric grid voltages is illustrated in a twodimensional cut view. The preservation of the duality of the staggered grids requires adding new nodes to the dual grid, as well as shifting existing nodes. This is shown in Fig. 4. In order to obtain a value for the new or shifted voltages, an interpolation procedure has to be carried out. Furthermore, it has to be distinguished between the interpolation of voltages oriented in parallel to the refinement [e.g. $\hat{e}_{x}^{\prime}\left(2^{\prime}\right)$ in Fig. 3(b)] and those oriented perpendicularly [e.g. $\hat{e}_{z}^{\prime}(2)$ and $\hat{e}_{z}^{\prime}\left(2^{\prime}\right)$ in Fig. 3(c)]. In the following, two interpolation procedures, linear and high order spline interpolation, will be discussed.
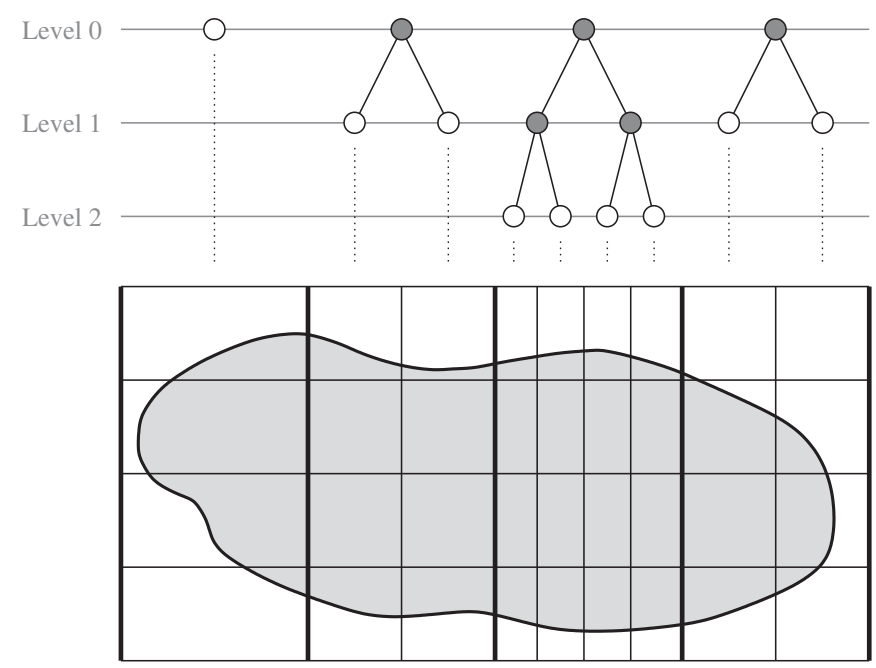

FIG. 2. Representation of the mesh refinement using consecutive bisections and the refinement levels. Nonrefined grid cells, such as the cells on the left end, have refinement level zero. The refinement history of a cell is recorded in a binary tree, with each bisection increasing the refinement level by one. The current grid is related to the tree leaves (white circles). Their depth in the tree reflects the refinement level $L$ with the grid step size reducing as $1 / 2^{L}$. 

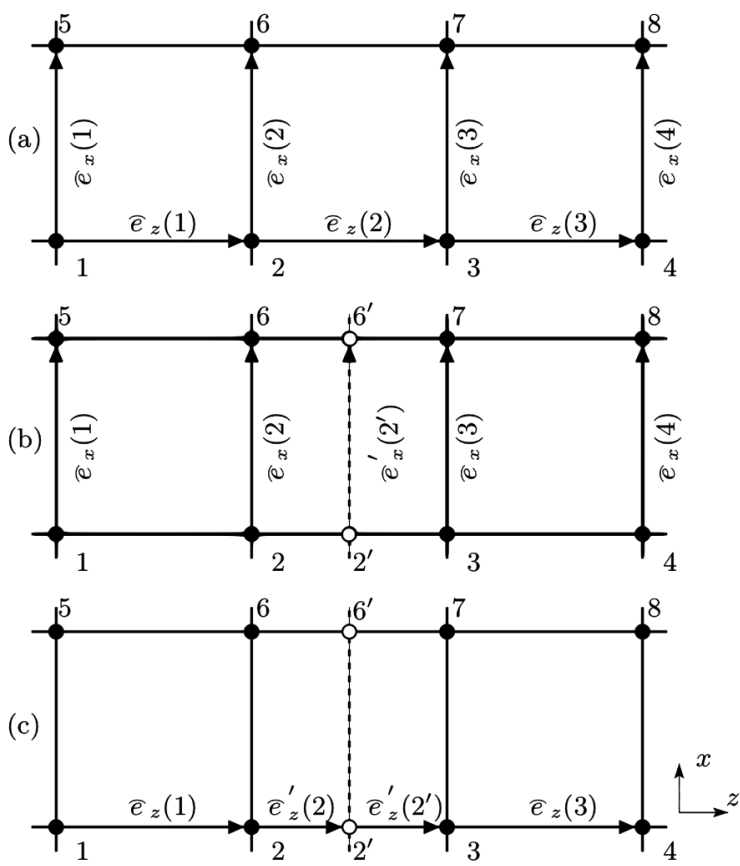

FIG. 3. The refinement of the primary grid and the assignment of electric grid voltages to the refined edges is depicted in the $x-z$ plane. In (a) the initial situation is shown. In (b) and (c) the middle cell was refined, introducing an additional grid line (dashed). Thus, two nodes $2^{\prime}$ and $6^{\prime}$ (circles) are inserted. All voltages that are new or require an update of their value are marked with a prime. In (b) the position of the new electric grid voltage $\hat{e}_{x}^{\prime}\left(2^{\prime}\right)$ is shown. The voltage $\hat{e}_{z}(2)$, oriented along the $z$ coordinate, has to be split into the two voltages $\hat{e}_{z}^{\prime}(2)$ and $\hat{e}_{z}^{\prime}\left(2^{\prime}\right)$ as shown in (c).

\section{A. Linear Interpolation of grid voltages}

The application of a linear interpolation for the determination of new or shifted voltages is straightforward. The interpolation procedure described below refers to the refinement scenario shown in Figs. 3 and 4. The lengths of the primary and dual grid edges [cf. (12)] are denoted by $|c|$ and $|\tilde{c}|$, respectively. Discrete field quantities associated with a specific node $i$ or $\tilde{i}$ are denoted as, e.g., $c(i)$. Primed quantities are either new, or they have to be recomputed during the adaptation procedure.

a. Refinement.-The value of new electric grid voltages oriented parallel to the refinement [e.g. $\left.\hat{e}_{x}^{\prime}\left(2^{\prime}\right)\right]$ is determined by

$$
\hat{e}_{x}^{\prime}\left(2^{\prime}\right)=\frac{\hat{e}_{x}(2)+\hat{e}(3)}{2} .
$$

The splitting of voltages oriented perpendicularly to the refinement $\left[\right.$ e.g. $\left.\hat{e}_{z}(2)\right]$ is constrained by the condition

$$
\hat{e}_{z}^{\prime}(2)+\hat{e}_{z}^{\prime}\left(2^{\prime}\right) \stackrel{!}{=} \hat{e}_{z}(2) \text {. }
$$

This condition imposes the conservation of the total voltage between nodes 2 and 3. A detailed sketch of this refinement is shown in Fig. 5. In order to assign values to (a)

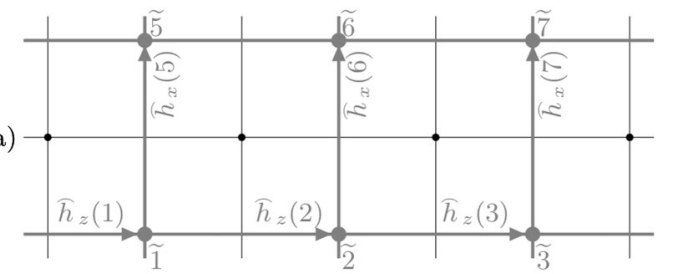

(b)

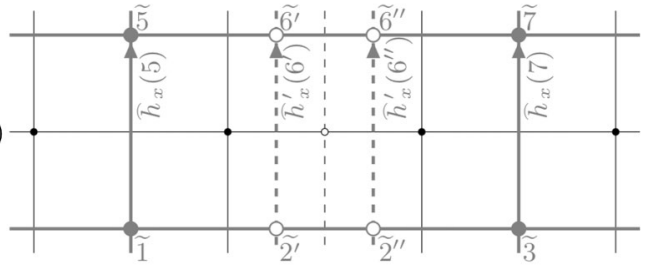

(c)

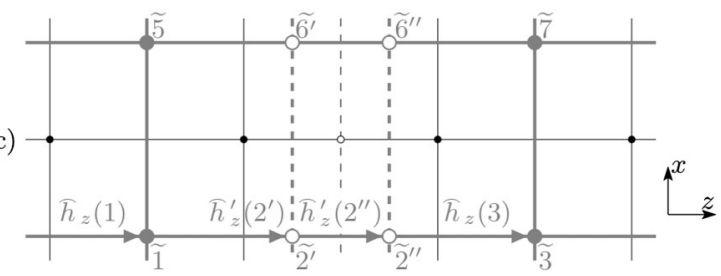

FIG. 4. The refinement of the dual grid (gray), imposed by the refined primary grid (thin black lines) is depicted. The initial situation is shown in (a). In order to preserve the duality of the two grids the dual nodes $\tilde{2}$ and $\tilde{6}$ have to be shifted. Their new position is indicated by $\tilde{2}^{\prime}$ and $\tilde{6}^{\prime}$ in (b) and (c). In addition, two new nodes, $\tilde{2}^{\prime \prime}$ and $\tilde{6}^{\prime \prime}$, have to be inserted. In (b) the assignment of $x$-oriented magnetic voltages is shown and in (c) the assignment of $z$-oriented voltages.

the refined voltages $\hat{e}_{z}^{\prime}(2)$ and $\hat{e}_{z}^{\prime}\left(2^{\prime}\right)$ the voltage gradient, i.e., the average electric field, along the axis is approximated by a central difference using the neighboring voltages $\hat{e}_{z}(1)$ and $\hat{e}_{z}(3)$. The voltages assigned to the refined edges read

$$
\hat{e}_{z}^{\prime}(2)=\left|c^{\prime}(2)\right|\left(e_{z}(2) \frac{\left|c^{\prime}(2)\right|}{2} \frac{\Delta e_{z}(3,1)}{\Delta z(\tilde{3}, \tilde{1})}\right)
$$

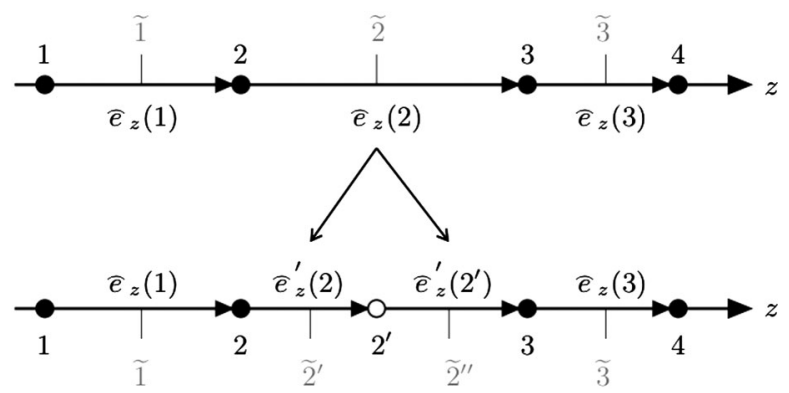

FIG. 5. Interpolation of electric grid voltages oriented perpendicularly to the refinement. The coarse grid voltage $\hat{e}_{z}(2)$ is split into the two voltages $\hat{e}_{z}^{\prime}(2)$ and $\hat{e}_{z}^{\prime}\left(2^{\prime}\right)$ such that the total voltage in between nodes 2 and 3 is preserved [cf. Eq. (25)]. In order to assign values to $\hat{e}_{z}^{\prime}(2)$ and $\hat{e}_{z}^{\prime}\left(2^{\prime}\right)$, the voltage gradient along the axis is evaluated using $\hat{e}_{z}(1)$ and $\hat{e}_{z}(3)$ [cf. Eqs. (26) and (27)]. 


$$
\hat{e}_{z}^{\prime}\left(2^{\prime}\right)=\left|c^{\prime}\left(2^{\prime}\right)\right|\left(e_{z}(2)+\frac{\left|c^{\prime}\left(2^{\prime}\right)\right|}{2} \frac{\Delta e_{z}(3,1)}{\Delta z(\tilde{3}, \tilde{1})}\right)
$$

where the sampled electric field variables $e_{z}(i)$ are introduced as

$$
e_{z}(i):=\frac{\hat{e}_{z}(i)}{|c(i)|}
$$

the $z$ coordinate of the node $i$ is denoted by $z(i)$, and the $\Delta$ notation denotes a difference of the respective quantity, e.g., $\Delta z(\tilde{3}, \tilde{1})=z(\tilde{3})-z(\tilde{1})$. Since cells are always divided into halves, $\left|c^{\prime}(2)\right|$ equals $\left|c^{\prime}\left(2^{\prime}\right)\right|$ and the condition (25) is fulfilled.

The interpolation of the magnetic voltages is more cumbersome. Besides the insertion of new dual grid nodes, also existing nodes have to be shifted in position in order to preserve the duality of the staggered grids. Shifting a node, however, implies a modification of the grid voltages along the associated edges.

The interpolation of magnetic voltages oriented in parallel to the refinement is illustrated in Fig. 4(b). The voltages $\hat{h}_{x}^{\prime}\left(\tilde{\sigma}^{\prime}\right)$ and $\hat{h}_{x}^{\prime}\left(\tilde{\sigma}^{\prime \prime}\right)$ are given by

$$
\begin{aligned}
& \hat{h}_{x}^{\prime}\left(\tilde{6}^{\prime}\right)=\hat{h}_{x}(\tilde{6})-\Delta z\left(\tilde{6}, \tilde{6}^{\prime}\right) \frac{\Delta \hat{h}_{x}(\tilde{7}, \tilde{5})}{\Delta z(\tilde{7}, \tilde{5})}, \\
& \hat{h}_{x}^{\prime}\left(\tilde{6}^{\prime \prime}\right)=\hat{h}_{x}(\tilde{6})+\Delta z\left(\tilde{6}^{\prime \prime}, \tilde{6}\right) \frac{\Delta \hat{h}_{x}(\tilde{7}, \tilde{5})}{\Delta z(\tilde{7}, \tilde{5})} .
\end{aligned}
$$

Similarly to the splitting of electric voltages, the splitting of magnetic voltages, oriented perpendicularly to the refinement, is constrained by the conservation of the total voltage. However, for a complete description of the refinement algorithm, the bisection of at least two neighboring cells has to be considered. This is illustrated in Fig. 6. For this case, the conservation condition for the total voltage between the nodes $\tilde{1}$ and $\tilde{4}$ can be expressed as

$$
\begin{aligned}
& \hat{h}_{z}^{\prime}\left(\tilde{2}^{\prime}\right)+\hat{h}_{z}^{\prime}\left(\tilde{2}^{\prime \prime}\right)+\hat{h}_{z}^{\prime}\left(\tilde{3}^{\prime}\right)+\hat{h}_{z}^{\prime}\left(\tilde{3}^{\prime \prime}\right)+\hat{h}_{z}^{\prime}(\tilde{4}) \\
& \quad \stackrel{!}{=} \hat{h}_{z}(\tilde{2})+\hat{h}_{z}(\tilde{3})+\hat{h}_{z}(\tilde{4}) .
\end{aligned}
$$

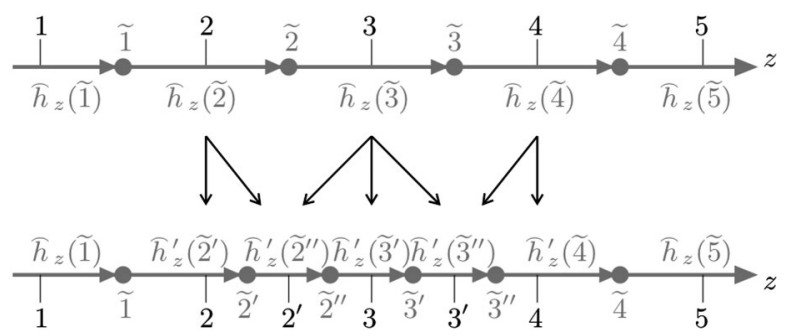

FIG. 6. Interpolation of magnetic grid voltages oriented perpendicularly to the refinement. The voltages $h_{z}(\tilde{2}), h_{z}(\tilde{3})$, and $h_{z}(\tilde{4})$, assigned to coarse grid edges, are split and distributed such that the total magnetic voltage in between the nodes $\tilde{2}$ and $\tilde{4}$ is preserved [cf. Eq. (31)]. The voltages on the refined grid are determined according to Eqs. (32)-(36).
The refined magnetic voltages read

$$
\begin{gathered}
\hat{h}_{z}^{\prime}\left(\tilde{2}^{\prime}\right)=\left|\tilde{c}^{\prime}\left(\tilde{2}^{\prime}\right)\right|\left[h_{z}(\tilde{2})-\left(z(2)-\frac{z(\tilde{1})+z\left(\tilde{2}^{\prime}\right)}{2}\right) \frac{\Delta h_{z}(\tilde{4}, \tilde{2})}{\Delta z(4,2)}\right], \\
\hat{h}_{z}^{\prime}\left(\tilde{2}^{\prime \prime}\right)=\Delta z\left(\tilde{2}, \tilde{2}^{\prime}\right) \cdot h_{z}(\tilde{2}),+\Delta z\left(\tilde{2}^{\prime \prime}, \tilde{2}\right) \cdot h_{z}(\tilde{3}), \\
\hat{h}_{z}^{\prime}\left(\tilde{3}^{\prime}\right)=\left|\tilde{c}^{\prime}\left(\tilde{3}^{\prime}\right)\right| \cdot h_{z}(\tilde{3}), \\
\hat{h}_{z}^{\prime}\left(\tilde{3}^{\prime \prime}\right)=\Delta z\left(\tilde{3}, \tilde{3}^{\prime}\right) \cdot h_{z}(\tilde{3})+\Delta z\left(\tilde{3}^{\prime \prime}, \tilde{3}\right) \cdot h_{z}(\tilde{4}), \\
\hat{h}_{z}^{\prime}(\tilde{4})=\left|\tilde{c}^{\prime}(\tilde{4})\right| \cdot\left[h_{z}(\tilde{4})+\left(\frac{z\left(\tilde{3}^{\prime \prime}\right)+z(\tilde{4})}{2}-z(4)\right) \cdot \frac{\Delta h_{z}(\tilde{4}, \tilde{2})}{\Delta z(4,2)}\right],
\end{gathered}
$$

with the sampled magnetic field $h_{z}(\tilde{i})$ defined as

$$
h_{z}(\tilde{i}):=\frac{\hat{h}_{z}(\tilde{i})}{|\tilde{c}(\tilde{i})|} .
$$

Since the following equations hold true,

$$
z(2)-\frac{z(\tilde{1})+z\left(\tilde{2}^{\prime}\right)}{2}=\frac{z\left(\tilde{3}^{\prime \prime}\right)+z(\tilde{4})}{2}-z(4),
$$

and

$$
\begin{gathered}
|\tilde{c}(\tilde{2})|=\left|\tilde{c}^{\prime}\left(\tilde{2}^{\prime}\right)\right|+\Delta z\left(\tilde{2}, \tilde{2}^{\prime}\right), \\
|\tilde{c}(\tilde{3})|=\Delta z\left(\tilde{2}^{\prime \prime}, \tilde{2}\right)+\left|\tilde{c}^{\prime}\left(\tilde{3}^{\prime}\right)\right|+\Delta z\left(\tilde{3}, \tilde{3}^{\prime}\right), \\
|\tilde{c}(\tilde{4})|=\Delta z\left(\tilde{3}^{\prime \prime}, \tilde{3}\right)+\left|\tilde{c}^{\prime}(\tilde{4})\right|,
\end{gathered}
$$

Eqs. (32)-(36) fulfill the condition (31).

Equations (24), (26), (27), (29), (30), and (32)-(36) define the rules for performing linear interpolations of the state variables $\hat{\mathbf{e}}$ and $\hat{\mathbf{h}}$. They enable consistent grid refinement based on cell bisection within the FIT framework.

b. Coarsening.-In the following, the rules for performing grid coarsening are given. We refer again to Figs. 3 and 4 and assume the removal of the nodes $2^{\prime}$ and $6^{\prime}$ and the associated dual nodes. Alongside with the removal of the primary nodes $2^{\prime}$ and $6^{\prime}$ and the connecting edge, the electric grid voltage $\hat{e}_{x}^{\prime}\left(2^{\prime}\right)$ is eliminated from the vector of electric voltages. No further modification is required for all electric voltages oriented in parallel to the refinement. The constraint (25) defines the rule for merging electric voltages oriented perpendicularly to the refinement,

$$
\hat{e}_{z}(2)=\hat{e}_{z}^{\prime}(2)+\hat{e}_{z}^{\prime}\left(2^{\prime}\right) .
$$

For the determination of the magnetic coarse grid voltage $\hat{h}_{x}(\tilde{6})$, oriented parallel to the refinement, a linear interpolation of the neighboring voltages allocated on the refined grid is performed: 


$$
\hat{h}_{x}(\tilde{6})=\frac{\hat{h}_{x}^{\prime}\left(\tilde{\sigma}^{\prime}\right)+\hat{h}_{x}^{\prime}\left(\tilde{6}^{\prime \prime}\right)}{2} .
$$

The magnetic coarse grid voltages, oriented perpendicularly to the refinement, are obtained by the summation of fractional voltages given on the fine grid (see Fig. 6). The merging of the fine grid magnetic voltages has to obey the constraint (31). The coarse grid voltages are given by

$$
\begin{gathered}
\hat{h}_{z}(\tilde{2})=\hat{h}_{z}^{\prime}\left(\tilde{2}^{\prime}\right)+\Delta z\left(\tilde{2}, \tilde{2}^{\prime}\right) \cdot h_{z}^{\prime}\left(\tilde{2}^{\prime \prime}\right), \\
\hat{h}_{z}(\tilde{3})=\Delta z\left(\tilde{2}^{\prime \prime}, \tilde{2}\right) \cdot h_{z}^{\prime}\left(\tilde{2}^{\prime \prime}\right)+\hat{h}_{z}^{\prime}\left(\tilde{3}^{\prime}\right)+\Delta z\left(\tilde{3}, \tilde{3}^{\prime}\right) \cdot h_{z}^{\prime}\left(\tilde{3}^{\prime \prime}\right),
\end{gathered}
$$

$$
\hat{h}_{z}(\tilde{4})=\Delta z\left(\tilde{3}^{\prime \prime}, \tilde{3}\right) h_{z}^{\prime}\left(\tilde{3}^{\prime \prime}\right)+\hat{h}_{z}^{\prime}(\tilde{4}) .
$$

Since the following holds true,

$$
\begin{aligned}
& \left|\tilde{c}\left(\tilde{2}^{\prime \prime}\right)\right|=\Delta z\left(\tilde{2}, \tilde{2}^{\prime}\right)+\Delta z\left(\tilde{2}^{\prime \prime}, \tilde{2}\right), \\
& \left|\tilde{c}\left(\tilde{3}^{\prime \prime}\right)\right|=\Delta z\left(\tilde{3}, \tilde{3}^{\prime}\right)+\Delta z\left(\tilde{3}^{\prime \prime}, \tilde{3}\right),
\end{aligned}
$$

the condition (31) is fulfilled also for the case of grid coarsening.

Equations (42)-(46) describe the required modifications of the FIT field quantities during the coarsening process of the computational grid. In combination with the refinement rules given above, they provide for a consistent framework for performing time-adaptive conformal grid refinement within the FIT. Refinement schemes other than by cell bisecting are generally possible. However, these are associated with a larger complexity in mesh administration as well as an additional decision about the cell division ratio and will not be considered in this work.

\section{B. Spline interpolation}

A linear interpolation of the discrete field quantities is easy to implement and fast in code execution. The interpolated quantities are second order accurate, which is in agreement with the theoretical order of accuracy of the FIT. However, applying higher order interpolating functions may be beneficial for obtaining a smoother representation of high-frequency fields on the refined mesh. We observed a positive effect of applying spline interpolations on the overall field smoothness.

The natural cubic spline (C-spline) is a common choice. It is required to pass through the given data points exactly, and to be twice continuously differentiable with the second derivative equal to zero on every interval boundary [8]. For its determination, a tridiagonal system of equations has to be solved. During a time-domain simulation adopting time-adaptive grid refinement, hundreds to thousands of grid adaptations are performed, each involving thousands of cells. Thus, the overall computational costs for solving even a small system of equations quickly become nonviable. In addition, the natural cubic spline may exhibit overshoots (see Fig. 7). Therefore, we apply the once continuously differentiable Akima subspline [9], which does not require solving a system of equations and exhibits reduced overshooting.

Cubic (sub)splines can conveniently be characterized by the triple of values $[x(i), f(i), s(i)]$ for each data point $i$, where $x(i)$ is the coordinate of the data point, $f(i)$ its value, and $s(i)$ its slope. Imposing the conditions

$$
\begin{aligned}
S[x(i-1)] & =f(i-1), & & S[x(i)]=f(i), \\
S^{\prime}[x(i-1)] & =s(i-1), & & S^{\prime}[x(i)]=s(i),
\end{aligned}
$$

at every interval boundary, uniquely describes the broken cubic spline function

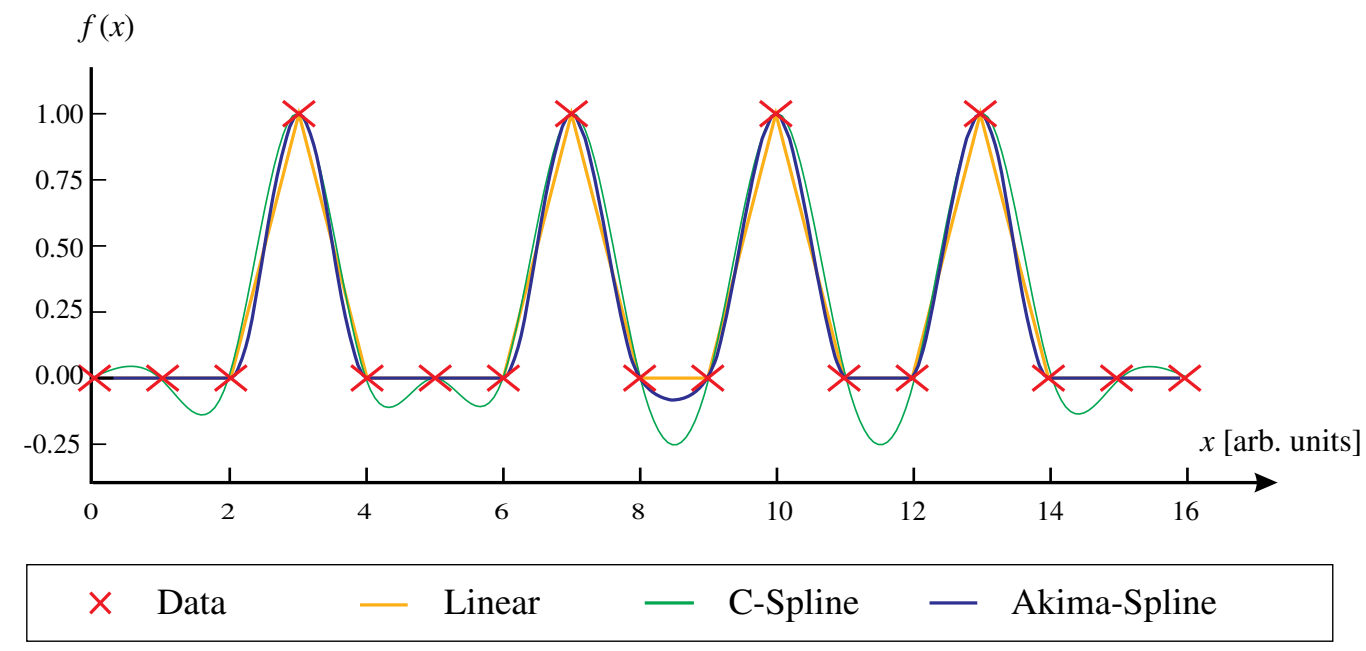

FIG. 7. Comparison of spline interpolations. The given data points are interpolated using linear interpolation, a natural cubic spline (C-spline), and an Akima spline. The C-spline exhibits an oscillatory behavior and strong overshooting. Up to the data point 10, Akima's original slope estimation was employed [cf. Eq. (50)]. From this point on the minmod slope limiter [cf. Eq. (51)] was applied, which effectively avoids overshooting. 


$$
\begin{aligned}
S(x)= & a_{0}+a_{1}[x-x(i-1)]+a_{2}[x-x(i-1)]^{2} \\
& +a_{3}[x-x(i-1)]^{3},
\end{aligned}
$$

with the coefficients

$$
\begin{aligned}
& a_{0}=f(i-1), \quad a_{1}=s(i-1), \\
& a_{2}=\frac{3}{(\Delta x)^{2}} \Delta f(i, i-1)-\frac{1}{\Delta x}[s(i)+2 s(i-1)], \\
& a_{3}=\frac{2}{(\Delta x)^{3}} \Delta f(i, i-1)+\frac{1}{(\Delta x)^{2}} \Delta s(i, i-1) .
\end{aligned}
$$

However, the slopes $s(i)$ are, in general, unknown. The Akima procedure makes use of a local heuristic method for estimating the slopes. It involves the data point $i$ and two neighboring points on each side. First, the piecewise gradients $g$, given by

$$
g(j):=\frac{f(j+1)-f(j)}{x(j+1)-x(j)}, \quad \text { with } j=i-2, \quad i-1, i, i+1,
$$

are evaluated. These are weighted with the factors $w$,

$$
\begin{aligned}
w(i-1) & :=|g(i+1)-g(i)|, \\
w(i) & :=|g(i-1)-g(i-2)|,
\end{aligned}
$$

yielding the estimated slope at data point $i$,

$$
s(i):=\frac{w(i-1) g(i-1)+w(i) g(i)}{w(i-1)+w(i)} .
$$

The fundamental idea of the heuristic can be summarized as: the larger the difference between the two gradients on one side of point $i$, the larger is the weighting factor applied to the gradient on the other side of this point. This is intended to minimize overshooting and oscillatory behavior. Note that Akima's heuristic is closely related to the idea of slope limiters. These are commonly used in finite volume methods for reconstructing a piecewise continuous solution on the grid (cf. [10]). Using, e.g., the minmod limiter the estimated slope reads

$$
\begin{aligned}
& s(i):=\operatorname{minmod}[g(i), g(i+1)] \\
& = \begin{cases}g(i), & \{\forall i|| g(i)|<| g(i+1) \mid, g(i) g(i+1)>0\}, \\
g(i+1), & \{\forall i|| g(i)|>| g(i+1) \mid, g(i) g(i+1)>0\}, \\
0, & \{\forall i \mid g(i) g(i+1) \leq 0\} .\end{cases}
\end{aligned}
$$

Out of its two arguments, the minmod operator chooses the smaller one if they are equally signed and zero otherwise. An overview of slope limiting techniques is found in [11].

In Fig. 7 the interpolation of a set of data points using linear interpolation the $\mathrm{C}$-spline, and the Akima spline is illustrated. The C-spline shows an oscillatory behavior and strong overshooting. For the determination of the Akima spline up to data point 10 , the slope was calculated using Akima's slope definition (50). For the points 11 to 16 the minmod limiter was applied, which effectively avoids any overshooting. Empirically, field smoothness was best when using slope-limited Akima splines with slopes according to (51).

The actual spline interpolation procedure of the discrete field quantities is illustrated in Fig. 8. In (a) and (b) the primary and dual grid nodes along the $z$ coordinate are indicated in black and gray. In (b) the refined area has moved by a distance of one grid cell into the $z$ direction. Therefore, the grid topology around cells 2 (coarsening) and 5 (refinement) has to be modified. The spline is set up using the position of the grid nodes and the allocated field data in the refined area and some neighboring cells. An evaluation of the spline function at the position of new or shifted nodes yields the interpolated field values.

(a)

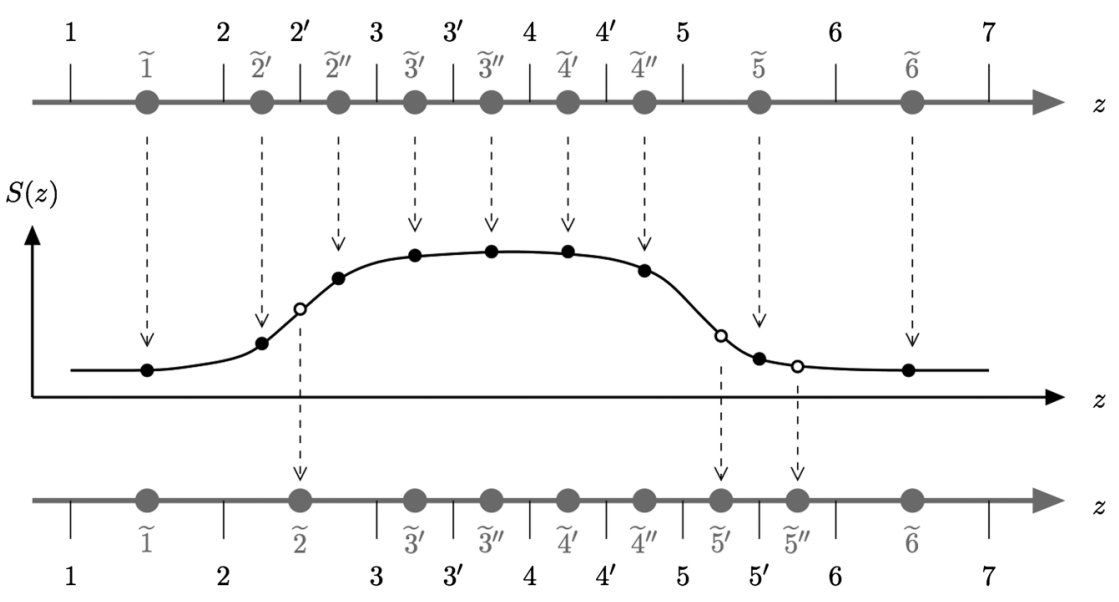

FIG. 8. Setup and evaluation of the interpolating spline. An exemplary arrangement of grid nodes along the $z$ coordinate is shown in (a) and (b). In (b) the refined area of the grid has moved by a distance of one grid cell into the $z$ direction. Hence, the grid has to be coarsened around node 2 and refined around node 5. The interpolating spline is set up, using the position of the nodes and field data in the refined area and some neighboring cells (black dots). An evaluation of the spline function at the position of new or shifted nodes (circles) yields the interpolated field values. 
The Akima and the slope-limited Akima splines are viable choices for performing interpolations within the computational grid. They are formally third order accurate and can be fully constructed from local information. Thus, they do not require the solution of a system of equations, which makes their implementation very efficient for the purpose of adaptive grid refinement.

\section{CHARGED PARTICLE SIMULATIONS ON ADAPTIVE GRIDS}

Simulations of the dynamics of charged particles using the FIT are performed routinely and have first been reported in 1988 in [12]. For self-consistent simulations the particle-in-cell (PIC) algorithm is applied [13]. It employs macroparticles, which may adopt any position from the space-continuous domain of interest. Macroparticles carry the charge and mass of about $10^{3-5}$ individual particles. Because of (11) the trajectory of a particle in the presence of electromagnetic fields depends only on the ratio of its charge and mass, making this a valid simplification.

The PIC algorithm consists of three steps. First, the electromagnetic field at the space-continuous position of each macroparticle has to be obtained from the spacediscrete FIT quantities. This is done by means of a trilinear interpolation. Next, the equations of motion (10) and (11) are integrated using the algorithm described in [14]. Finally, the convective currents are calculated from the position increment of all particles. For this last step we adapted the cloud-in-cell (CIC) approach $[15,16]$ to work with time-adaptive mesh refinement. The particles are modeled as a uniformly charged volume of finite extent, i.e., a charged cloud.

The extent of the cloud is usually chosen to coincide with the size of the cells. However, employing nonequidistant grids or adaptive grid refinement gives rise to nonuniform cell sizes, which demands for modification of the algorithm. There are two modification options: first, a constant size of the particle cloud is chosen independently from the grid cell size or, second, the size of the cloud is adapted in order to match the sizes of the cells involved. Depending on the local degree of grid refinement, the first option can largely increase the number of cells affected by one cloud. Besides the coding efforts coming along with this nonlocal operation, the deposit of fractional currents to a large number of grid points increases the computational load. Therefore, the second option has been pursued. The adaptation of the cloud in dependence of the grid cell size is illustrated in Fig. 9 for a one-dimensional example.

The modified CIC approach maintains its charge conserving property, meaning that it fulfills the discrete continuity equation (23) in a cell-wise manner.

\section{APPLICATIONS}

In this section, we present results of the application of the dynamic mesh adaptation algorithm introduced above to the simulation of two setups.

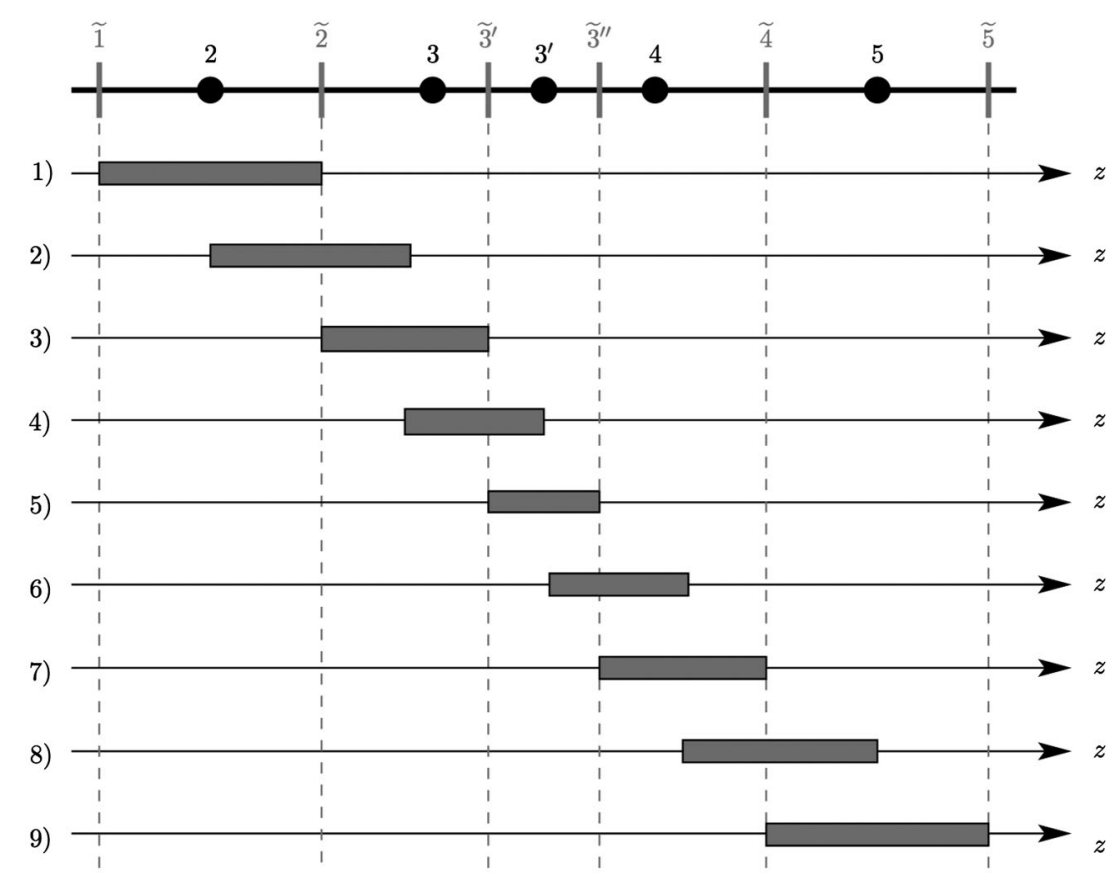

FIG. 9. Illustration of the adaptation of the particle cloud size in one dimension. The size of the particle cloud relates to the extent of the covered cells on the dual grid. If the particle is centered within a dual grid cell (cases 1, 3, 5, 7, and 9), the sizes of the cloud and the dual volume coincide. Otherwise, the cloud has to be adapted asymmetrically around the particle position. For cases 2 and 4 the charge density within the part of the cloud in the dual volumes $\tilde{2}$ or $\tilde{3}^{\prime}$ has to be increased as a consequence of the diminished size. Contrarily, for cases 6 and 8, the charge density of the cloud in the dual volumes $\tilde{3}^{\prime \prime}$ and $\tilde{4}$ has to be decreased. 
TABLE I. Settings of the benchmark example.

\begin{tabular}{lcccc}
\hline \hline Pipe length & Pipe radius & RMS bunch radius & RMS bunch length & Particle velocity \\
\hline $120 \mathrm{~mm}$ & $40 \mathrm{~mm}$ & $5 \mathrm{~mm}$ & $3 \mathrm{~mm}$ & $0.9 c_{0}$ \\
\hline \hline
\end{tabular}

\section{A. Bunch drift in a pipe}

In this example we are concerned with a comparison of the accuracy of numerical solutions obtained using adaptive and nonadaptive meshes. As the example is small, the savings in terms of computational costs from applying adaptive mesh refinement are rather small as well. Large savings are achieved in the second example in Sec. VIB.

We consider a hollow, perfectly conducting pipe, which is closed by a perfectly conducting plate at one end. The other end is left open. A bunch of charged particles is emitted from the center of the end plate and travels along the pipe at a constant velocity of $0.9 c_{0}$, where $c_{0}$ is the speed of light in vacuum. The settings of the computational model are specified in Table I. The analytical solution of this problem for a semi-infinite pipe is given in [17].

In the simulations the axis of the cylinder is aligned with the $z$ coordinate. In Figs. 10(a) and 10(b), the analytical solution of the longitudinal electric field along the axis is depicted by a black curve. The FIT and LT-FIT solutions are shown in red and green, respectively. For the results shown in Fig. 10(a), an equidistant grid was applied. In

(a)

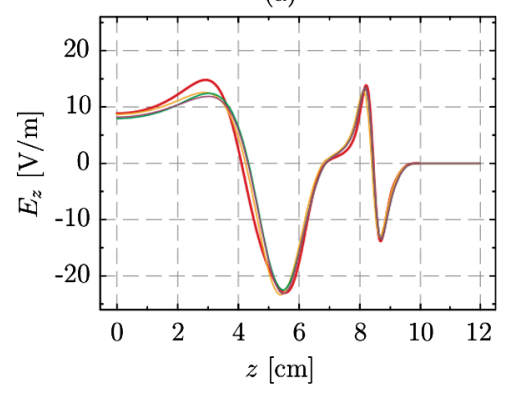

(c)

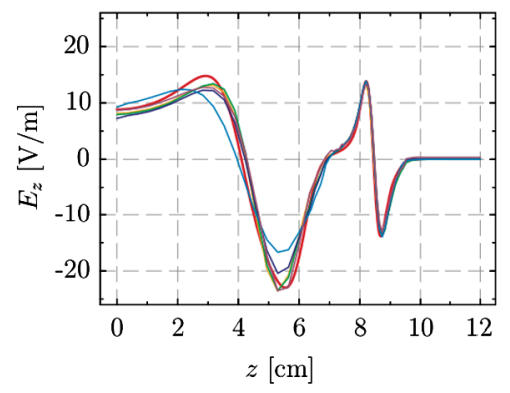

Fig. 10(b) the errors, given by the pointwise Euclidean distance of the analytical and the respective numerical solution, are plotted. The results obtained using dynamic mesh refinement $(L=3)$ in the bunch region are shown in Fig. 10(c) and the respective errors in Fig. 10(d).

A series of simulations of this test setup using various mesh and mesh refinement settings has been carried out. The results are presented in Tables II (FIT) and III (LT-FIT). The tables list the respective grid information, i.e., the refinement level $L$, the number of grid points in the nonrefined base grid, $N_{x}$ and $N_{z}$, the transverse grid step size, $\Delta x$, the minimum longitudinal step size $\Delta z_{\min }$, and the number of degrees of freedom (DoF). For the adaptive simulations, the number of DoF corresponds to the mean value. The tables list as well the computation time in seconds, the relative $L^{2}$ error, and the total variation (TV) of the longitudinal electric field along the cylinder axis. The latter two are measures for the quality of the numerical solutions. The relative $L^{2}$ error is computed as

$$
\mathcal{E}^{\mathrm{rel}}=\frac{\left\|\mathbf{E}_{z}-\mathbf{e}_{z}\right\|_{2}}{\left\|\mathbf{E}_{z}\right\|_{2}}
$$

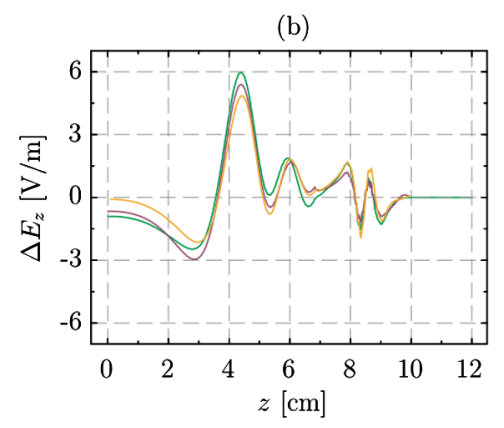

(d)
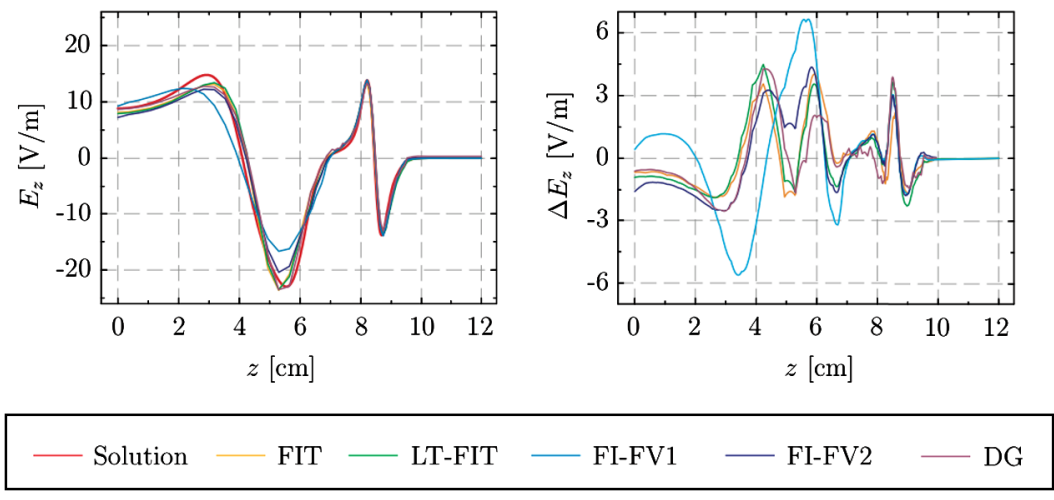

FIG. 10. Longitudinal component of the electric field, excited by a Gaussian bunch traveling in a pipe. The black curve in (a) and (c) indicates the analytical solution for the setup described in Table I. All other curves show results obtained from numerical simulations. In the top row, a static, equidistant computational grid was applied. The results are shown in (a), their errors in (b). In the bottom row, time-adaptive grid refinement $(L=3)$ was applied. In (c) the results for this case are shown, in (d) the errors. 
TABLE II. Results of FIT simulations: The top data row lists results obtained on a static, equidistant grid. Below, results obtained on time-adaptive grids using slope-limited Akima splines are displayed.

\begin{tabular}{|c|c|c|c|c|c|}
\hline \multicolumn{6}{|c|}{$L N_{x} N_{z} \Delta x / \mathrm{mm} \Delta z_{\min } / \mathrm{mm} \mathrm{DoF} / 1 \times 10^{6} \mathrm{Time} / \mathrm{sec} \quad \mathcal{E}^{\mathrm{rel}} \mathrm{TV}$} \\
\hline \multicolumn{6}{|l|}{ Static grid } \\
\hline 0135210 & 0.59 & 0.57 & 22.96 & 2066 & 0.0411 .00 \\
\hline \multicolumn{6}{|c|}{ Time-adaptively refined grid } \\
\hline 1135105 & 0.59 & 0.57 & 13.00 & 1225 & 0.0441 .42 \\
\hline 213552 & 0.59 & 0.58 & 9.00 & 940 & 0.0401 .93 \\
\hline 313527 & 0.59 & 0.56 & 8.50 & 925 & 0.0411 .99 \\
\hline 413513 & 0.59 & 0.58 & 11.50 & 1025 & 0.0391 .55 \\
\hline
\end{tabular}

TABLE III. Results of LT-FIT simulations: The top data row lists results obtained on a static, equidistant grid. Below, results obtained on time-adaptive grids using slope-limited Akima splines are displayed.

\begin{tabular}{|c|c|c|c|c|c|}
\hline \multicolumn{6}{|c|}{$L N_{x} N_{z} \Delta x / \mathrm{mm} \Delta z_{\min } / \mathrm{mm} \mathrm{DoF} / 1 \times 10^{6}$ Time $/ \mathrm{sec} \quad \mathcal{E}^{\text {rel }} \quad \mathrm{TV}$} \\
\hline \multicolumn{6}{|l|}{ Static grid } \\
\hline 0135210 & 0.59 & 0.57 & 22.96 & 3543 & 0.0380 .63 \\
\hline \multicolumn{6}{|c|}{ Time-adaptively refined grid } \\
\hline 1135105 & 0.59 & 0.57 & 13.00 & 2008 & 0.0451 .39 \\
\hline 213552 & 0.59 & 0.58 & 9.00 & 1443 & 0.0411 .65 \\
\hline 313527 & 0.59 & 0.56 & 8.50 & 1447 & 0.0401 .80 \\
\hline 413513 & 0.59 & 0.58 & 11.50 & 1791 & 0.0371 .40 \\
\hline
\end{tabular}

with $\mathbf{E}_{z}=\left(E_{z}[z(1)], \ldots, E_{z}\left[z\left(N_{z}\right)\right]\right)^{\mathrm{T}}$ and $E_{z}$ the longitudinal component of the analytical solution of the electric field at the grid point positions $z\left(i_{z}\right)$.

The total variation is a measure for the smoothness of a function [18]. It is defined as

$$
T V[E(z)]:=\int_{z(1)}^{z\left(N_{z}\right)}\left|E^{\prime}(z)\right| d z .
$$

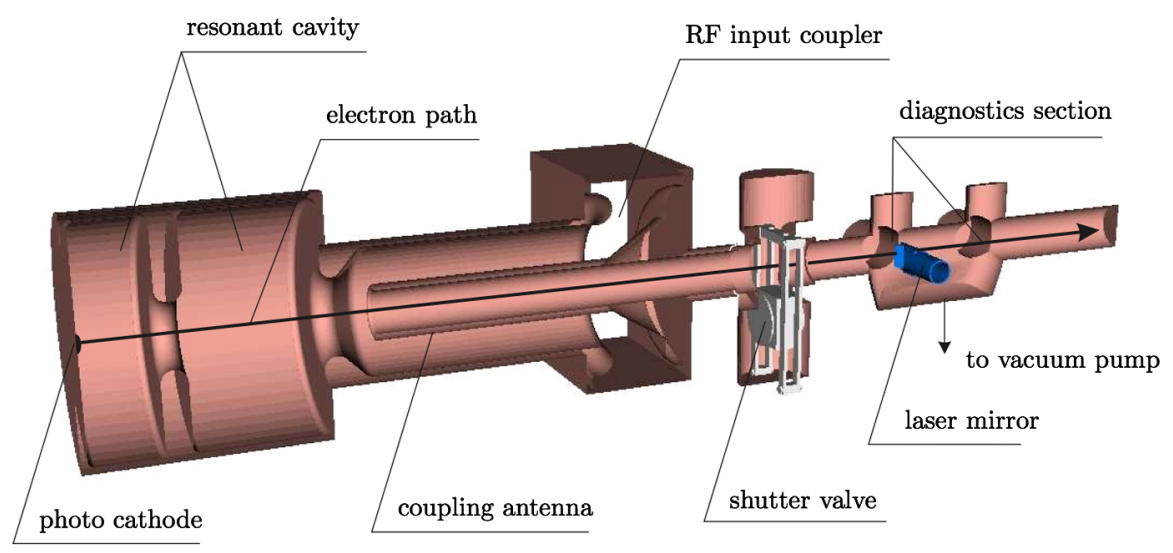

FIG. 11. A CAD model of the injector section of the Free Electron Laser in Hamburg (FLASH) is shown in cut view. The depicted part corresponds to approximately $60 \mathrm{~cm}$ of the injector. In total $2 \mathrm{~m}$ have been modeled. However, the nondepicted part consists only of the beam pipe. A short laser pulse is directed onto the photocathode, where electrons are emitted. The bunch of electrons is accelerated by a high-frequency electromagnetic field, which is excited in the resonant cavity. It propagates along the electron path through the coupling antenna and passes the shutter valve and the laser mirror, which is inserted through one arm of the diagnostics section. The other arms are used for inserting measurement devices. The opening on its bottom side is connected to a vacuum pump.
In [19] it is shown that the total variation of a function equals the sum of local minima and maxima, where the values at the integration end points count once and all other extrema count twice. The total variation is, therefore, a direct measure for oscillations and their amplitudes. For discrete solutions it is computed as

$$
T V\left(\mathbf{e}_{z}\right)=\sum_{i_{z}=1}^{N_{z}-1}\left|e_{z}\left(i_{z}+1\right)-e_{z}\left(i_{z}\right)\right| .
$$

The standard FIT is a very well established method for performing PIC simulations. All total variation values are, hence, normalized to the value obtained with the FIT on the finest, nonadaptive grid. For comparability, the settings were chosen such that all simulations finish within less than $1 \mathrm{~h}$.

A comparison of the results shows that: (i) for identical mesh resolutions in the bunch region the adaptive simulations yield errors similar to those obtained on nonadaptive meshes. Hence, the accuracy of the solutions for this example is mainly determined by the resolution in the bunch region. (i) For this example adaptive mesh refinement saves about one half of the degrees of freedom and computing time. (iii) The relative errors of the FIT and the LT-FIT are similar, however, the total variation of the LT-FIT solution is significantly lower, indicating reduced oscillations due to better numerical dispersion properties. (iv) For identical numbers of DoF the LT-FIT simulations take longer because of the higher computational costs for the time integration.

\section{B. Self-consistent simulation of the PITZ rf gun}

In order to drive a free-electron-laser (FEL) operating by the self-amplified spontaneous-emission (SASE) principle, highly charged electron beams of high brightness are 
TABLE IV. PITZ design parameters.

\begin{tabular}{lc}
\hline \hline Parameter & Design value \\
\hline Bunch charge & $1 \mathrm{nC}$ \\
Transverse laser profile & Hat profile \\
Laser spot radius & $1 \mathrm{~mm}$ \\
Longitudinal laser profile & Flattop \\
Laser pulse duration & $22 \mathrm{ps}$ \\
Rise/fall time & $2 \mathrm{ps}$ \\
Accelerating gradient & $42 \mathrm{MV} / \mathrm{m}$ \\
Transverse emittance & $1 \mathrm{~mm} \mathrm{mrad}$ \\
\hline \hline
\end{tabular}

required [20]. The aim of the PITZ project (Photo Injector Test Facility at DESY Zeuthen) [21] is the development and testing of an injector capable of delivering such high quality beams. The injectors for the Free Electron Laser in Hamburg (FLASH) [22] and the future European X-Ray Laser Project XFEL [23] are under development at PITZ.

The layout of the radio-frequency (rf) gun of the injector is shown in Fig. 11. The emitted bunch is accelerated in a 1.5-cell L-band cavity providing for an accelerating gradient of $42 \mathrm{MV} / \mathrm{m}$ at an operational frequency of $1.3 \mathrm{GHz}$. A focusing technique proposed in [24] is applied in order to compensate for the correlated part of the space-charge induced emittance growth. The gun was designed such that a minimum of the transverse emittance is expected at a distance of approximately $1.6 \mathrm{~m}$ downstream of the cathode. At this position a rf cavity is installed in order to accelerate the electrons to relativistic energies. The main design parameters are listed in Table IV.

Many numerical studies of the injector using PIC codes have been performed over the past years. Results have been published, e.g., in [25-29]. However, these simulations either assumed a rotationally symmetric geometry and made use of a so-called 2.5-dimensional approach as implemented in the MAFIA TS2 module [30], or otherwise very short distances in the cm-range of the full threedimensional model were simulated. Results of parallelized simulations of the full three-dimensional model up to $1 \mathrm{~m}$ downstream of the cathode have been presented in [4,31].
However, the exact position of the transverse emittance minimum is required for the optimal placement of the subsequent accelerating cavity. Also, the value of the emittance at this position is of strong interest for checking whether the design value is met.

In the context of this work, the gun was simulated up to a distance of two meters downstream of the cathode. First, settings of the simulation parameter such as the grid resolution and the number of macroparticles have been determined. Then, a design study was performed, which addresses the effects of individual injector elements on the beam quality.

Previous investigations have shown that the length of the simulated bunches critically depends on the longitudinal grid step size [32]. In [25] it was stated that a grid resolution of $20 \mu \mathrm{m}$ longitudinally is required. In Fig. 12(a) the results of a parameter study addressing the relation of the longitudinal grid step size and the computed bunch length are presented. The initial $2.5 \mathrm{~cm}$ of the gun were simulated using an equidistant grid of 2.5 to $80 \mu \mathrm{m}$ step size. The computed root-mean-square (RMS) bunch length $\sigma_{z}$ at $z=2.5 \mathrm{~cm}$ varies from 2.24 to $2.35 \mathrm{~mm}$. Changing the step size from 5 to $2.5 \mu \mathrm{m}$ results in a variation of $\sigma_{z}$ by approximately $1 \mu \mathrm{m}$. The computed bunch lengths for a step size of 2.5 and $10 \mu \mathrm{m}$ differ by approximately $10 \mu \mathrm{m}$. A longitudinal grid step of $10 \mu \mathrm{m}$ is considered to be a reasonable balance of computational costs and accuracy.

After emission the electrons have a very low energy of approximately $5 \mathrm{eV}$ and space-charge forces have a strong influence. Since the particles gain energy quickly, it is sufficient to apply the smallest grid step size only in the immediate vicinity of the cathode. Hence, an additional static grid refinement is applied in this area. In Fig. 12(b) results for this approach are shown. A grid with a uniform step size of $80 \mu \mathrm{m}$ is statically refined within the first centimeter from the cathode. Refinement levels from one to four have been applied, resulting in step sizes of 40,20, 10 , and $5 \mu \mathrm{m}$ within the refined region. The results are, except for minor discrepancies, identical to those obtained with an equidistant grid of the same minimum step size.
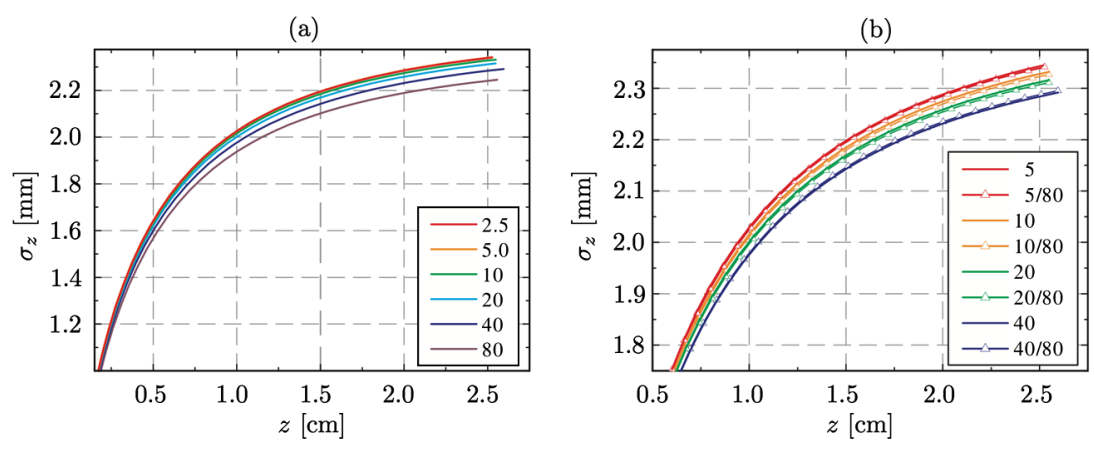

FIG. 12. Computed RMS bunch length vs longitudinal grid step size. In (a) equidistant grids with different grid step sizes were used. In (b) the equidistant grid with a step size of $80 \mu \mathrm{m}$ was statically refined within the first cm from the cathode. The step sizes given in the legend are in units of $\mu \mathrm{m}$. 
(a)

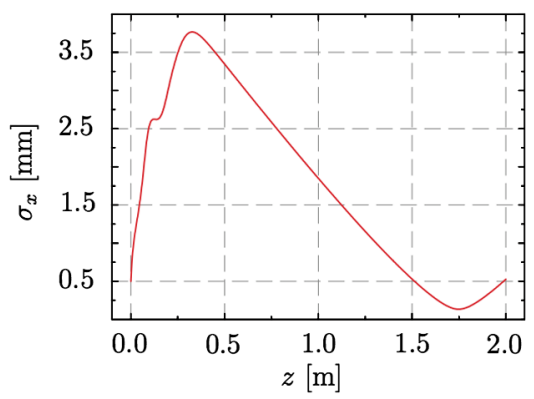

(c)

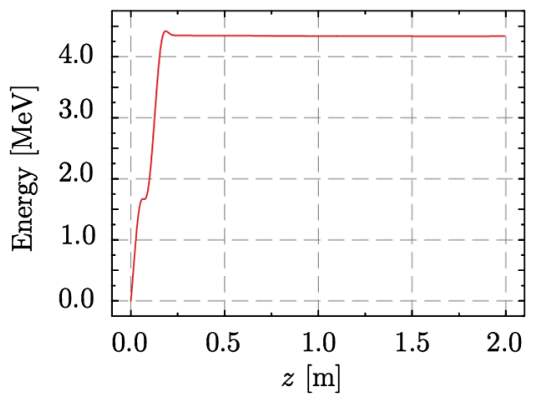

(b)

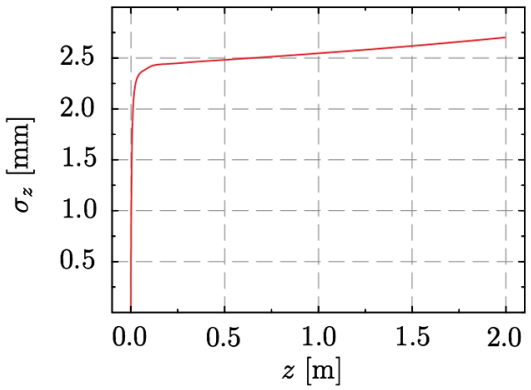

(d)

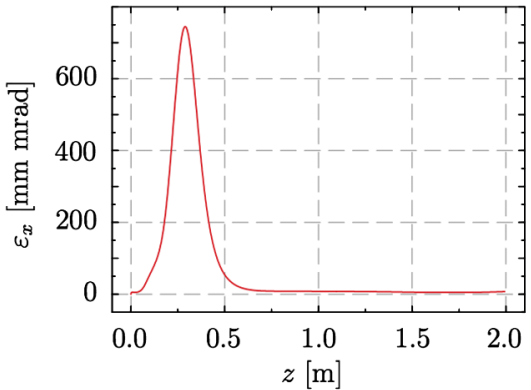

FIG. 13. The plots show the evolution of the RMS bunch width (a), RMS bunch length (b), average particle energy (c), and horizontal emittance (d) along the longitudinal coordinate of the PITZ rf gun.

(a)

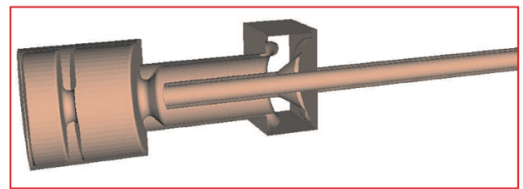

(c)

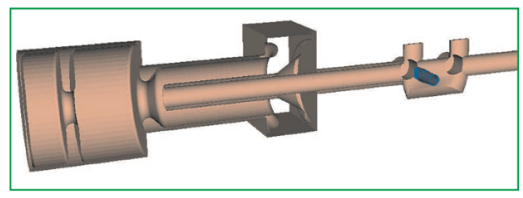

(e)

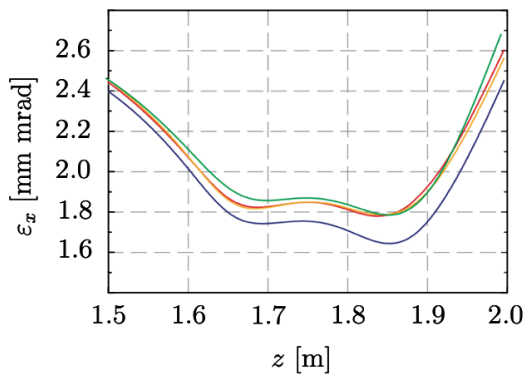

(b)

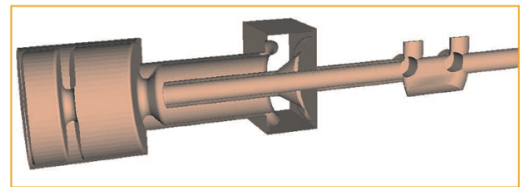

(d)

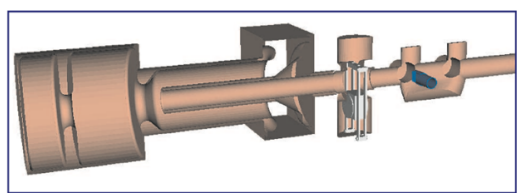

(f)

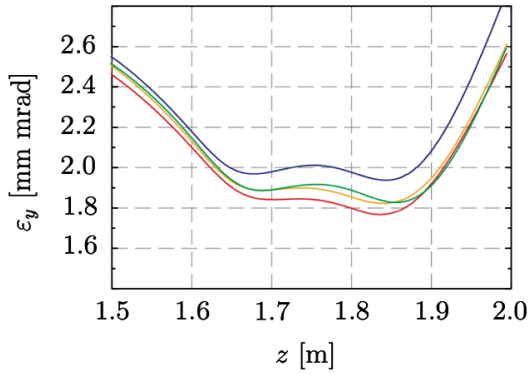

+ + Valve

FIG. 14. Models and results of the PITZ rf gun design study. In (a) to (d) the CAD models of the simulated structures are shown. The horizontal and vertical emittances around the position of their minimum are plotted in (e) and (f), respectively. The colors of the curves correspond to the outline color of the models above. While $\varepsilon_{x}$ and $\varepsilon_{y}$ are similar for case (a), they show a distinct asymmetry for the model depicted in (d). 
For the simulation of the full structure, a combination of static and dynamic mesh refinement was applied providing for a longitudinal resolution of $10 \mu \mathrm{m}$ for the first $\mathrm{cm}$ from the cathode and $80 \mu \mathrm{m}$ thereafter. The requirements on the transverse grid step size are less demanding. The width of the simulated bunches using a step size of 2.5 and $80 \mu \mathrm{m}$ differ by less than $5 \mu \mathrm{m}$. Deviations in the $\mu \mathrm{m}$ range correspond to some per mille of the bunch width. Choosing a minimum transverse step size of $80 \mu \mathrm{m}$ and a refinement level of five, we obtained an adaptive computational mesh consisting of an average of approximately $82 \times 10^{6}$ cells. A nonadaptive mesh providing for the same minimum resolution would consist of approximately $1.7 \times 10^{9}$ cells. The adaptive approach, thus, reduces the number of cells by a factor larger than 20, making the simulation of the problem feasible on a single workstation. The bunches in the simulations comprised approximately $0.5 \times 10^{6}$ macroparticles.

The results given in the following were obtained with the LT-FIT method. In Fig. 13, the evolution of the horizontal root-mean-square (RMS) bunch width $\sigma_{x}$ (a), the RMS bunch length $\sigma_{z}$ (b), the average particle energy (c), and the horizontal emittance $\varepsilon_{x}$ (d) for the model shown in Fig. 11 are plotted. The projected emittances $\varepsilon_{x}$ and $\varepsilon_{y}$ are given by $\varepsilon_{u}=\left[\sigma_{u}^{2} \cdot \sigma_{p_{u}}^{2}-\left(\sigma_{u, p_{u}}\right)^{2}\right]^{12}, u \in\{x, y\}$, where $\sigma_{p_{u}}^{2}$ is the momentum variance and $\sigma_{u, p_{u}}$ is the covariance of position and momentum.

We performed a design study of the injector in order to identify the individual effects of the diagnostics section, the laser mirror, and the shutter valve on the beam quality in terms of emittance growth. The respective models are shown in Figs. 14(a)-14(d). For each model, one more element is added. The colors of the curves in Figs. 14(e) and 14(f) correspond to the outline color of the respective model.

For the model given in Fig. 14(a), the horizontal and vertical emittances, $\varepsilon_{x}$ and $\varepsilon_{y}$, are identical. This is expected since the geometry is rotationally symmetric for all parts close to the beam. The rf input coupler is obviously not rotationally symmetric but this symmetry violation is hidden by the coupling antenna. In Fig. 14(b), the large opening on the bottom side of the doublecross introduces an asymmetry of the geometry in the horizontal and the vertical direction. While $\varepsilon_{x}$ is almost unchanged, the value of $\varepsilon_{y}$ increases by approximately $0.04 \mathrm{~mm}$ mrad. The laser mirror, added in the horizontal plane, introduces another asymmetry [cf. Fig. 14(c)]. An influence on the transverse emittances can be identified in the results but the actual emittance growth at the position of the minimum is small. Finally, the shutter valve depicted in Fig. 14(d) results in significant changes of the transverse emittances. While the horizontal emittance actually decreases by approximately $0.1 \mathrm{~mm} \mathrm{mrad}$, the vertical emittance increases by about $0.18 \mathrm{~mm} \mathrm{mrad}$ in comparison to the structure of Fig. 14(a). Hence, the shutter valve causes a distinct asymmetry in the transverse beam dynamics. In the meantime, a shutter valve with rf shieldings has been installed.

\section{CONCLUSIONS}

In this paper, we extended the framework of Finite Integration Technique to include dynamic mesh refinement. This offers computability on workstations for a class of multiscale problems, which before was accessible to massively parallelized computations only. In particular, our approach enabled us to perform fully self-consistent simulations of the PITZ rf gun up to two meters downstream from the cathode. We elaborated on the details concerning the mesh refinement and coarsening procedures and presented a novel type of subspline interpolation, which is entirely devoid of overshooting. Finally, we performed a design study, which identifies the emittance growth due to individual parts of the gun.

\section{ACKNOWLEDGMENTS}

The work of S. Schnepp is supported by the "Initiative for Excellence" of the German Federal and State Governments and the Graduate School of Computational Engineering at Technische Universität Darmstadt. The authors thank Thomas Lau for providing the analytical solution to the example used in Sec. VI A.

[1] F. Wolfheimer, E. Gjonaj, and T. Weiland, Nucl. Instrum. Methods Phys. Res., Sect. A 558, 202 (2006).

[2] C. K. Birdsall, Plasma Physics via Computer Simulation (Institute of Physics, London, 1991).

[3] T. Weiland, Part. Accel. 15, 245 (1984).

[4] E. Gjonaj, T. Lau, S. Schnepp, F. Wolfheimer, and T. Weiland, New J. Phys. 8, 285 (2006).

[5] T. Weiland, Part. Accel. 17, 227 (1985).

[6] G. Strang, SIAM J. Numer. Anal. 5, 506 (1968).

[7] T. Lau, E. Gjonaj, and T. Weiland, Frequenz 59, 210 (2005).

[8] C. de Boor, A Practical Guide to Splines (Springer, New York, 2001).

[9] H. Akima, J. ACM 17, 589 (1970).

[10] B. Van Leer, Towards the Ultimate Conservative Scheme I. The Quest of Monotonicity, Lectures Notes in Physics (Springer, New York, 1973), pp. 163-168.

[11] R. J. LeVeque, Numerical Methods for Conservation Laws (Birkhaeuser, Basel, Switzerland, 1990).

[12] W. Bialowons, M. Bieler, H.-D. Bremer, F.-J. Decker, H.-C. Lewin, P. Schuett, G.-A. Voss, R. Wanzenberg, and T. Weiland, in Proceedings of the 1st European Particle Accelerator Conference (EPAC), Rome, Italy (1988), Vol. 1, pp. 902-904.

[13] C. K. Birdsall, IEEE Trans. Plasma Sci. 19, 65 (1991).

[14] J. Boris, in Proceedings of the 4th Conference on Numerical Simulation of Plasmas (1970).

[15] C. K. Birdsall and D. Fuss, J. Comput. Phys. 3, 494 (1969). 
[16] J. Villasenor and O. Buneman, Comput. Phys. Commun. 69, 306 (1992).

[17] I. N. Onishchenko, D. Y. Sidorenko, and G. V. Sotnikov, Phys. Rev. E 65, 066501 (2002).

[18] A. Harten, J. Comput. Phys. 49, 357 (1983).

[19] C.B. Laney, Computational Gasdynamics (Cambridge University Press, Cambridge, UK, 1998).

[20] Z. Huang and K.-J. Kim, Phys. Rev. ST Accel. Beams 10, 034801 (2007).

[21] F. Stephan, K. Abrahamyan, G. Asova, J. Bähr, G. Dimitrov, U. Gensch, H.-J. Grabosch, J. Han, M. Krassilnikov, D. Lipka, V. Miltchev, A. Oppelt, B. Petrosyan, D. Pose, S. Riemann, L. Staykov, M. von Hartrott, E. Jaeschke, D. Krämer, C. Richter, I. Bohnet, J.-P. Carneiro, K. Flöttmann, S. Schreiber, J. Rossbach, P. Michelato, L. Monaco, C. Pagani, D. Sertore, I. Tsakov, W. Sandner, I. Will, W. Ackermann, W. Müller, S. Schnepp, S. Setzer, and T. Weiland, in Proceedings of the 26th International Free Electron Laser Conference (Sincrotrone Trieste S.C.p.A, Trieste, Italy, 2004), pp. 347-350.

[22] W. Ackermann, G. Asova, V. Ayvazyan, A. Azima, N. Baboi, J. Baehr, V. Balandin, B. Beutner, A. Brandt, A. Bolzmann, R. Brinkmann, O. I. Brovko, M. Castellano, P. Castro, L. Catani, E. Chiadroni, S. Choroba, A. Cianchi, J. T. Costello, D. Cubaynes, J. Dardis, W. Decking, H. Delsim-Hashemi, A. Delserieys, G. D. Pirro, M. Dohlus, S. Duesterer, A. Eckhardt, H. T. Edwards, B. Faatz, J. Feldhaus, K. Floettmann, J. Frisch, L. Froehlich, T. Garvey, U. Gensch, C. Gerth, M. Goerler, N. Golubeva, H.-J. Grabosch, M. Grecki, O. Grimm, K. Hacker, U. Hahn, J. H. Han, K. Honkavaara, T. Hott, M. Huening, Y. Ivanisenko, E. Jaeschke, W. Jalmuzna, T. Jezynski, R. Kammering, V. Katalev, K. Kavanagh, E. T. Kennedy, S. Khodyachykh, K. Klose, V. Kocharyan, M. Koerfer, M. Kollewe, W. Koprek, S. Korepanov, D. Kostin, M. Krassilnikov, G. Kube, M. Kuhlmann, C. L. S. Lewis, L. Lilje, T. Limberg, D. Lipka, F. Loehl, H. Luna, M. Luong, M. Martins, M. Meyer, P. Michelato, V. Miltchev, W. D. Moeller, L. Monaco, W. F. O. Mueller, O. Napieralski, O. Napoly, P. Nicolosi, D. Noelle, T. Nu?ez, A. Oppelt, C. Pagani, R. Paparella, N. Pchalek, J. Pedregosa-Gutierrez, B. Petersen, B. Petrosyan, G. Petrosyan, L. Petrosyan, J. Pflueger, E. Ploenjes, L. Poletto, K. Pozniak, E. Prat, D. Proch, P. Pucyk, P. Radcliffe, H. Redlin, K. Rehlich, M. Richter, M. Roehrs, J. Roensch, R. Romaniuk, M. Ross, J.
Rossbach, V. Rybnikov, M. Sachwitz, E. L. Saldin, W. Sandner, H. Schlarb, B. Schmidt, M. Schmitz, P. Schmueser, J.R. Schneider, E. A. Schneidmiller, S. Schnepp, S. Schreiber, M. Seidel, D. Sertore, A. V. Shabunov, C. Simon, S. Simrock, E. Sombrowski, A. A. Sorokin, P. Spanknebel, R. Spesyvtsev, L. Staykov, B. Steffen, F. Stephan, F. Stulle, H. Thom, K. Tiedtke, M. Tischer, S. Toleikis, R. Treusch, D. Trines, I. Tsakov, E. Vogel, T. W. H. Weise, M. Wellhoefer, M. Wendt, I. Will, A. Winter, K. Wittenburg, W. Wurth, P. Yeates, M. V. Yurkov, I. Zagorodnov, and K. Zapfe, Nature Photon. 1, 336 (2007).

[23] M. Altarelli, R. Brinkmann, M. Chergui, W. Decking, B. Dobson, S. Duesterer, G. Gruebel, W. Graeff, H. Graafsma, J. Hajdu, J. Marangos, J. Pflueger, H. Redlin, D. Riley, I. Robinson, J. Rossbach, A. Schwarz, K. Tiedtke, T. Tschentscher, I. Vartaniants, H. Wabnitz, H. Weise, R. Wichmann, K. Witte, A. Wolf, M. Wulff, and M. Yurkov, The European X-Ray Free-Electron LaserTechnical design report (2007).

[24] B. Carlston, Nucl. Instrum. Methods Phys. Res., Sect. A 285, 313 (1989).

[25] M. Zhang and P. Schuett, in Proceedings of the Computational Accelerator Physics Conference (CAP) (1996) [http://proceedings.aip.org/resource/2/apcpcs/391/ 1/21_1].

[26] R. Cee, M. Krassilnikov, S. Setzer, T. Weiland, and A. Novokhatski, Nucl. Instrum. Methods Phys. Res., Sect. A 483, 321 (2002).

[27] S. Setzer, W. Ackermann, S. Schnepp, and T. Weiland, in Proceedings of the 9th European Particle Accelerator Conference (EPAC) (Lucerne, 2004), pp. 1981-1983.

[28] S. Schnepp, E. Gjonaj, and T. Weiland, in Proceedings of the 10th European Particle Accelerator Conference (EPAC) (Edinburgh, Scotland, 2006), pp. 2182-2184.

[29] S. Schnepp, E. Gjonaj, and T. Weiland, in Proceedings of the 2007 Particle Accelerator Conference, Albuquerque, New Mexico (IEEE, New York, 2007).

[30] CST AG, Bad Nauheimer Str. 19, 64289 Darmstadt, Germany.

[31] F. Wolfheimer, E. Gjonaj, and T. Weiland, in Proc. of the 9th International Computational Accelerator Physics Conference (ICAP), Chamonix, France (2006).

[32] S. Schnepp, E. Gjonaj, and T. Weiland, in Proceedings of the 9th International Computational Accelerator Physics Conference (ICAP) (Ref. [32]). 